\title{
SUMSETS AND DIFFERENCE SETS CONTAINING A COMMON TERM OF A SEQUENCE
}

\author{
QUAN-HUI YANG and YONG-GAO CHEN ${ }^{\circledR}$
}

(Received 28 April 2011)

\begin{abstract}
Let $\beta>1$ be a real number, and let $\left\{a_{k}\right\}$ be an unbounded sequence of positive integers such that $a_{k+1} / a_{k} \leq \beta$ for all $k \geq 1$. The following result is proved: if $n$ is an integer with $n>(1+1 /(2 \beta)) a_{1}$ and $A$ is a subset of $\{0,1, \ldots, n\}$ with $|A| \geq(1-1 /(2 \beta+1)) n+\frac{1}{2}$, then $(A+A) \cap(A-A)$ contains a term of $\left\{a_{k}\right\}$. The lower bound for $|A|$ is optimal. Beyond these, we also prove that if $n \geq 3$ is an integer and $A$ is a subset of $\{0,1, \ldots, n\}$ with $|A|>\frac{4}{5} n$, then $(A+A) \cap(A-A)$ contains a power of 2. Furthermore, $\frac{4}{5}$ cannot be improved.
\end{abstract}

2010 Mathematics subject classification: primary 11B13; secondary 11B83, $05 \mathrm{D} 05$.

Keywords and phrases: sumsets, difference sets, sequences.

\section{Introduction}

Erdős and Freiman [3] proved a conjecture of Erdős and Freud: if $A \subseteq[1, n]$ with $|A|>n / 3$, then some power of 2 is the sum of elements of $A$. Later, Nathanson and Sárközy [7] proved that 3504 summands are enough. Freiman [4] reduced 3504 to 6. Finally, Lev [6] reduced 6 to 4. Here 4 is best possible (see Alon [2]). The key to Lev's proof is the following lemma: let $A \subseteq[0, n]$ with $|A| \geq \frac{1}{2} n+1$. Then either $A$ contains a power of 2, or there exist two distinct elements of $A$ whose sum is a power of 2 . Abe [1] and Pan [8] extended this result to the powers of an integer $m$.

For a set $A$ of integers, let $A+A=\left\{a_{1}+a_{2}: a_{1}, a_{2} \in A\right\}$ and $A-A=\left\{a_{1}-a_{2}\right.$ : $\left.a_{1}, a_{2} \in A\right\}$. Recently, Kapoor [5] extended the above results to general sequences. He proved that for an unbounded sequence $\left\{a_{k}\right\}$ of positive integers with $a_{k+1} / a_{k} \rightarrow \alpha$ as $k \rightarrow \infty$, and $\beta>\max (\alpha, 2)$, if $A \subset[0, x]$ is a set of integers with $0 \in A$ and

$$
|A| \geq\left(1-\frac{1}{\beta}\right) x,
$$

then $A+A$ contains a term of the sequence $\left\{a_{k}\right\}$.

This work was supported by the National Natural Science Foundation of China, Grant No. 11071121.

(C) 2011 Australian Mathematical Publishing Association Inc. 0004-9727/2011 \$16.00 
In this paper, the following result is proved.

THEOREM 1.1. Let $\beta>1$ be a real number, and let $\left\{a_{k}\right\}$ be an unbounded sequence of positive integers such that $a_{k+1} / a_{k} \leq \beta$ for all $k \geq 1$. Suppose that $n$ is an integer with $n>(1+1 /(2 \beta)) a_{1}$ and $A$ is a subset of $\{0,1, \ldots, n\}$.

(i) If all $a_{k}$ are even, and

$$
|A|>\left(1-\frac{1}{2 \beta+1}\right) n,
$$

then $(A+A) \cap(A-A)$ contains a term of $\left\{a_{k}\right\}$.

(ii) If $a_{k}$ are not all even, and

$$
|A| \geq\left(1-\frac{1}{2 \beta+1}\right) n+\frac{1}{2},
$$

then $(A+A) \cap(A-A)$ contains a term of $\left\{a_{k}\right\}$.

Furthermore (i) and (ii) are sharp.

From Theorem 1.1, by taking $a_{k}=2^{k}$ and $\beta=2$, we have the following corollary.

Corollary 1.2. Let $n \geq 3$ be an integer and $A$ be a subset of $\{0,1, \ldots, n\}$ such that $|A|>\frac{4}{5} n$. Then $(A+A) \cap(A-A)$ contains a power of 2 . Furthermore, $\frac{4}{5}$ cannot be improved.

\section{Proof of the theorem}

Let $k$ be the least integer such that

$$
n<\left(1+\frac{1}{2 \beta}\right) a_{k+1} .
$$

Then $k \geq 1$ and

$$
n \geq\left(1+\frac{1}{2 \beta}\right) a_{k} .
$$

We will show that $a_{k} \in(A+A) \cap(A-A)$.

First we prove that $a_{k} \in A-A$. Suppose that $a_{k} \notin A-A$.

Case 2.1. $(1+(1 / 2 \beta)) a_{k} \leq n<2 a_{k}$.

Since $a_{k}=\left(a_{k}+i\right)-i$ for $i=0,1, \ldots, n-a_{k}$, we have $\left|A \cap\left\{a_{k}+i, i\right\}\right| \leq 1$ for each $i \in\left\{0,1, \ldots, n-a_{k}\right\}$. Hence

$$
|A| \leq n+1-\left(n-a_{k}+1\right)=a_{k} \leq\left(1-\frac{1}{2 \beta+1}\right) n,
$$

a contradiction.

Case 2.2. $2 a_{k} \leq n<(1+(1 / 2 \beta)) a_{k+1}$. 
Since $a_{k}=\left(a_{k}+i\right)-i$ for $i=0,1, \ldots, a_{k}-1$, we have $\left|A \cap\left\{a_{k}+i, i\right\}\right| \leq 1$ for each $i \in\left\{0,1, \ldots, a_{k}-1\right\}$. Hence $|A| \leq n+1-a_{k}$.

If $a_{k}=1$ (this is a special case of (ii)), then

$$
n<\left(1+\frac{1}{2 \beta}\right) a_{k+1} \leq\left(1+\frac{1}{2 \beta}\right) \beta=\beta+\frac{1}{2} .
$$

Thus

$$
|A| \leq n+1-a_{k}=n<\left(1-\frac{1}{2 \beta+1}\right) n+\frac{1}{2}
$$

a contradiction.

If $a_{k} \geq 2$, then by

$$
n<\left(1+\frac{1}{2 \beta}\right) a_{k+1} \leq(2 \beta+1) \frac{1}{2} a_{k} \leq(2 \beta+1)\left(a_{k}-1\right),
$$

we have

$$
a_{k}-1>\frac{1}{2 \beta+1} n
$$

Thus

$$
|A| \leq n+1-a_{k}<n-\frac{1}{2 \beta+1} n=\left(1-\frac{1}{2 \beta+1}\right) n,
$$

a contradiction.

Now we prove that $a_{k} \in A+A$. Suppose that $a_{k} \notin A+A$.

Case 2.3. $a_{k}$ is even.

Since $a_{k}=\left(\frac{1}{2} a_{k}-i\right)+\left(\frac{1}{2} a_{k}+i\right)$ for $i=0,1, \ldots, \frac{1}{2} a_{k}$, we have $\frac{1}{2} a_{k} \notin A$ and so $\left|A \cap\left\{\frac{1}{2} a_{k}-i, \frac{1}{2} a_{k}+i\right\}\right| \leq 1$ for $i=1,2, \ldots, \frac{1}{2} a_{k}$. Hence $|A| \leq n+1-\frac{1}{2} a_{k}-1=n-$ $\frac{1}{2} a_{k}$. By

$$
n<\left(1+\frac{1}{2 \beta}\right) a_{k+1} \leq(2 \beta+1) \frac{1}{2} a_{k},
$$

we have

$$
|A| \leq n-\frac{1}{2} a_{k}<\left(1-\frac{1}{2 \beta+1}\right) n,
$$

a contradiction.

Case 2.4. $a_{k}$ is odd.

Since $a_{k}=\left(\frac{1}{2}\left(a_{k}-1\right)-i\right)+\left(\frac{1}{2}\left(a_{k}+1\right)+i\right)$ for $i=0,1, \ldots, \frac{1}{2}\left(a_{k}-1\right)$, we have $\left|A \cap\left\{\frac{1}{2}\left(a_{k}-1\right)-i, \frac{1}{2}\left(a_{k}+1\right)+i\right\}\right| \leq 1$ for $i=0,1, \ldots, \frac{1}{2}\left(a_{k}-1\right)$. Hence $|A| \leq n+1-$ $\frac{1}{2}\left(a_{k}+1\right)=n-\frac{1}{2} a_{k}+\frac{1}{2}$. By

$$
n<\left(1+\frac{1}{2 \beta}\right) a_{k+1} \leq(2 \beta+1) \frac{1}{2} a_{k},
$$


we have

$$
|A| \leq n-\frac{1}{2} a_{k}+\frac{1}{2}<\left(1-\frac{1}{2 \beta+1}\right) n+\frac{1}{2}
$$

a contradiction. We have proved (i) and (ii).

Next we show that Theorem 1.1 is sharp by taking $a_{k}=m^{k}$ for a fixed integer $m \geq 2$. In this case $\beta=m$. It is clear that

$$
|A| \geq\left(1-\frac{1}{2 m+1}\right) n+\frac{1}{2}
$$

is equivalent to

$$
|A|>\left(1-\frac{1}{2 m+1}\right) n+\frac{1}{2}-\frac{1}{4 m+2} .
$$

Let $\mathbb{N}$ denote the set of nonnegative integers. For odd $m \geq 3$, let $n=(2 m+1) m^{k} / 2-$ $1 / 2$ and $A=\left[m^{k} / 2+1 / 2,(2 m+1) m^{k} / 2-1 / 2\right] \cap \mathbb{N}$, where $k$ is a nonnegative integer. Then

$$
|A|=m^{k+1}=\left(1-\frac{1}{2 m+1}\right)\left(\frac{(2 m+1) m^{k}}{2}-\frac{1}{2}\right)+\frac{1}{2}-\frac{1}{4 m+2},
$$

so that (2.1) does not hold. Since $(A-A) \cap \mathbb{N} \subseteq\left[0, m^{k+1}-1\right]$ and $A+A \subseteq\left[m^{k}+1\right.$, $\left.(2 m+1) m^{k}-1\right]$, we have $(A+A) \cap(A-A) \subseteq\left[m^{k}+1, m^{k+1}-1\right]$, which contains no power of $m$.

For even $m \geq 2$, let $n=(2 m+1) m^{k} / 2$ and $A=\left[m^{k} / 2+1,(2 m+1) m^{k} / 2\right] \cap \mathbb{N}$, where $k$ is a nonnegative integer. Then

$$
|A|=m^{k+1}=\left(1-\frac{1}{2 m+1}\right) \frac{(2 m+1) m^{k}}{2},
$$

so the bound in case (i) does not hold. It follows that $(A-A) \cap \mathbb{N} \subseteq\left[0, m^{k+1}-1\right]$ and $A+A \subseteq\left[m^{k}+2,(2 m+1) m^{k}\right]$. Hence $(A+A) \cap(A-A) \subseteq\left[m^{k}+2, m^{k+1}-1\right]$, which also contains no power of $m$. This completes the proof of Theorem 1.1.

\section{Acknowledgement}

We would like to thank the referee for his/her comments.

\section{References}

[1] T. Abe, 'Sumsets containing powers of an integer', Combinatorica 24 (2004), 1-4.

[2] N. Alon, 'Subset sums', J. Number Theory 27 (1987), 196-205.

[3] P. Erdős and G. Freiman, 'On two additive problems', J. Number Theory 34 (1990), 1-12.

[4] G. A. Freiman, 'Sumsets and powers of 2', Colloq. Math. Soc. János Bolyai 60 (1992), 279-286.

[5] V. Kapoor, 'Sets whose sumset avoids a thin sequence', J. Number Theory 130 (2010), 534-538.

[6] V. F. Lev, 'Representing powers of 2 by a sum of four integers', Combinatorica 16 (1996), 413-416.

[7] M. B. Nathanson and A. Sárközy, 'Sumsets containing long arithmetic progressions and powers of 2', Acta Arith. 54 (1989), 147-154.

[8] H. Pan, 'Note on integer powers in sumsets', J. Number Theory 117 (2006), 216-221. 
QUAN-HUI YANG, School of Mathematical Sciences and Institute of Mathematics, Nanjing Normal University, Nanjing 210046, PR China

YONG-GAO CHEN, School of Mathematical Sciences and Institute of Mathematics, Nanjing Normal University, Nanjing 210046, PR China

e-mail:ygchen@njnu.edu.cn 